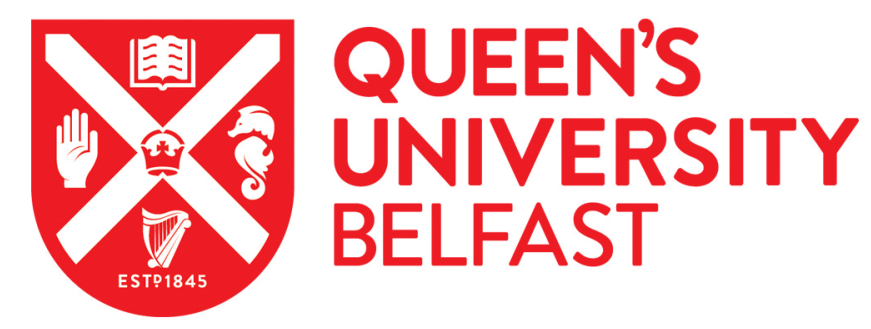

\title{
Spread OFDM-IM with precoding matrix and low-complexity detection designs
}

Luong, T. V., \& Ko, Y. (2018). Spread OFDM-IM with precoding matrix and low-complexity detection designs. IEEE Transactions on Vehicular Technology, 67(12), 11619 - 11626. https://doi.org/10.1109/TVT.2018.2872873

\author{
Published in: \\ IEEE Transactions on Vehicular Technology
}

Document Version:

Peer reviewed version

Queen's University Belfast - Research Portal:

Link to publication record in Queen's University Belfast Research Portal

Publisher rights

Copyright 2018 IEEE. This work is made available online in accordance with the publisher's policies. Please refer to any applicable terms of use of the publisher.

\section{General rights}

Copyright for the publications made accessible via the Queen's University Belfast Research Portal is retained by the author(s) and / or other copyright owners and it is a condition of accessing these publications that users recognise and abide by the legal requirements associated with these rights.

Take down policy

The Research Portal is Queen's institutional repository that provides access to Queen's research output. Every effort has been made to ensure that content in the Research Portal does not infringe any person's rights, or applicable UK laws. If you discover content in the Research Portal that you believe breaches copyright or violates any law, please contact openaccess@qub.ac.uk. 


\title{
Spread OFDM-IM with Precoding Matrix and Low-Complexity Detection Designs
}

\author{
Thien Van Luong, Student Member, IEEE, and Youngwook Ko, Senior Member, IEEE
}

\begin{abstract}
We propose a new spread Orthogonal Frequency Division Multiplexing with Index Modulation (S-OFDM-IM), which employs precoding matrices such as Walsh-Hadamard (WH) and Zadoff-Chu (ZC) to spread both non-zero data symbols of active sub-carriers and their indices, and then compress them into all available sub-carriers. This aims to increase the transmit diversity, exploiting both multipath and index diversities. As for the performance analysis, we derive the bit error probability (BEP) to provide an insight into the diversity and coding gains, and especially impacts of selecting various spreading matrices on these gains. This interestingly reveals an opportunity of using rotated versions of original $\mathrm{WH}$ and $\mathrm{ZC}$ matrices to further improve the BEP performance. More specifically, rotated matrices can enable S-OFDM-IM to harvest the maximum diversity gain which is the number of sub-carriers, while benchmark schemes have diversity gains limited by two. Moreover, we propose three low-complexity detectors, namely minimum mean square error log-likelihood ratio (MMSE-LLR), index pattern MMSE (IPMMSE), and enhanced IP-MMSE, which achieve different levels of complexity and reliability. Simulation results are presented to prove the superiority of S-OFDM-IM over the benchmarks.
\end{abstract}

Index Terms-OFDM-IM, index modulation, spreading, precoding, Zadoff-Chu, Walsh-Hadamard, MMSE-based detectors.

\section{INTRODUCTION}

Index modulation (IM) is an emerging technique that exploits indices of active channels to convey information in addition to conventional $M$-ary modulation symbols. The idea of IM is first proposed to code division multiplexing access systems in [1], where the index set of spread sequences is used to convey extra bits. Then, the IM concept is applied to the orthogonal frequency division multiplexing (OFDM) [2], [3], which results in a novel scheme termed as OFDM with index modulation (OFDM-IM). In particular, OFDM-IM activates only a subset of sub-carriers to carry data bits via both $M$-ary complex data symbols and active sub-carrier indices. Thus, this scheme provides a balanced trade-off between spectral efficiency (SE) and reliability, just by adjusting the number of active sub-carriers. Besides, OFDM-IM achieves higher energy efficiency (EE) and reliability than classical OFDM.

Recently, OFDM-IM has attracted a great deal of attention from researchers as shown in recent surveys [4], [5]. For example, in [6], the number of active sub-carriers is relaxed

Copyright (c) 2015 IEEE. Personal use of this material is permitted. However, permission to use this material for any other purposes must be obtained from the IEEE by sending a request to pubs-permissions@ieee.org.

This work was supported by the Engineering and Physical Sciences Research Council [grant number EP/N509541/1].

The authors are with the ECIT Institute, Queen's University Belfast, Belfast, BT3 9DT, UK, (e-mail: \{tluong01,y.ko\}@qub.ac.uk). to increase index bits. The greedy detector for OFDM-IM is proposed in [7] and its bit error rate (BER) under uncertain channel state information (CSI) is analyzed in [8]. In [9], the achievable rate is investigated, while a tight bound on the BER of the maximum likelihood (ML) detector is derived in [10]. The application of OFDM-IM in multiple input multiple output (MIMO) systems can be found in [11]. A novel framework of analyzing the symbol error probability under CSI uncertainty of OFDM-IM with any detection types is proposed in [12]. To improve the SE, in [6], the IM is separately applied to inphase and quadrature components to double the number of index bits and the resulting scheme is called as OFDM-IMI/Q. Dual-mode OFDM-IM and its extension to multi-mode and transmit diversity are reported in [13], [14], which exploit inactive sub-carriers to carry extra bits.

A variety of advanced schemes have been proposed to increase the transmit diversity of OFDM-IM. For instance, in [15], the coordinate interleaved OFDM-IM (CI-OFDM-IM) is proposed to convey real and imaginary parts of each non-zero data symbol over different sub-carriers. In [16], a compressed sensing-aided OFDM-IM is proposed to enhance the EE and diversity gain at the cost of higher complexity. Based on the code index modulation [17], the index modulated OFDM spread spectrum (IM-OFDM-SS) is introduced in [18], which uses indices of spreading codes to convey data bits. The use of coding and transmit diversity for OFDM-IM can be found in [19]. The repetition code for $M$-ary modulated symbols in OFDM-IM is presented in [20]. Meanwhile, the precoding or spreading technique has been well developed for classical OFDM. For example, the grouped linear constellation precoder (LCP) is optimally designed for OFDM [21] to provide maximum multipath diversity gains. In [22], the spread OFDM (SOFDM) employs the rotated Walsh-Hadamard (WH) transform to maximize diversity gain. Recently, the LCP is developed for OFDM-IM-I/Q [23] in order to increase the transmit diversity up to two. In [24], OFDM with grouped sub-carriers and the interleaving at the real dimension level is proposed, where the lattice-based codebook is designed to maximize the signal space diversity. In [25], the precoding matrix is designed for MIMO-OFDM-IM based on CSI at the transmitter. To the best of our knowledge, potentials of a precoded OFDM-IM to fully exploit both multipath and index diversities have been overlooked in the literature.

In this paper, we propose a novel IM scheme, which can maximize the transmit diversity of OFDM-IM. The contributions of this paper are summarized as follows:

- The spread OFDM-IM (S-OFDM-IM) is proposed, which employs WH and Zadoff-Chu (ZF) precoding matrices 
to spread both data symbols of active sub-carriers and their indices, and then compress them to all available sub-carriers. Thus, S-OFDM-IM can fully exploit both multipath and index diversities to substantially increase the transmit diversity.

- The bit error probability (BEP) is analyzed to provide an insight into diversity and coding gains, and effects of various spreading matrices on performance gains. This particularly sheds light on an opportunity of using phaseshifted spreading matrices of $\mathrm{WH}$ and $\mathrm{ZC}$ to possibly maximize the diversity order.

- Three low-complexity detectors are proposed, which offer different levels of reliability and complexity. The complexity analysis and comparison among detector types clearly show that complexities of the proposed detectors are substantially reduced over the ML.

- Extensive simulations are provided to show the superiority of S-OFDM-IM over its benchmarks. It is shown that despite using lower complexity detectors, our scheme still outperforms the benchmarks with the ML.

The rest of the paper is organized as follows. Section II describes the S-OFDM-IM system model with the WH and ZC spreading matrices. The performance analysis and the rotated spreading matrices are performed in Section III. In Section IV, we introduce low-complexity detectors. Simulation results are given in Section V, while Section VI concludes the paper. ${ }^{1}$

\section{SySTEM ModeL}

\section{A. S-OFDM-IM}

Consider an OFDM-IM system which has a total of $N_{c}$ sub-carriers. To ease implementation, these sub-carriers are partitioned into $G$ clusters of $N$ sub-carriers, i.e., $N_{c}=N G$ and the OFDM-IM process is independently performed in each cluster. In every transmission, only $K$ out of $N$ sub-carriers are active to carry information bits via not only $M$-ary modulated symbols but also active sub-carrier indices. Due to the fact that each cluster operates independently, without loss of generality, we present only one cluster hereinafter for simplicity.

We propose a new precoded IM scheme called as spread OFDM-IM (S-OFDM-IM), where active sub-carrier indices are spread and then compressed to all $N$ available sub-carriers. In particular, the block diagram of one S-OFDM-IM cluster is depicted in Fig. 1. For each transmission, $p$ incoming bits are divided into two bit streams $\left(p=p_{1}+p_{2}\right)$. The first $p_{1}$ bits are to determine a pattern of $K$ active indices, which is denoted by $\theta=\left\{i_{1}, \ldots, i_{K}\right\}$, where $i_{k} \in\{1, \ldots, N\}$. This mapping process is performed using look-up table or combinatorial method [3]. The remaining $p_{2}$ bits are mapped to $K$ complex data symbols denoted by $\mathbf{s}=\left[s_{1}, \ldots, s_{K}\right]$ with $s_{k} \in \mathcal{S}$, where $\mathcal{S}$ is the $M$-ary modulation constellation. The average energy per non-zero data symbol is normalized as $\mathbb{E}\left\{\left|s_{k}\right|^{2}\right\}=1$. Then,

\footnotetext{
${ }^{1}$ Notation: Upper-case bold and lower-case bold letters denote matrices and vectors, respectively. $(.)^{*},(.)^{T}$ and $(.)^{H}$ represent the complex conjugation, transpose and Hermitian operators, respectively. $\|$.$\| and \otimes$ denote the Frobenius norm and the Kronecker product, respectively. $j$ is the unit imaginary number. The binomial coefficient is denoted by $C($,$) , while the floor function$ is presented by $[.] . \mathcal{C N}($,$) denotes the complex Gaussian distribution. \mathbb{E}\{$. and $\mathcal{O}($.$) denote the average value and the Big-O notation, respectively.$
}

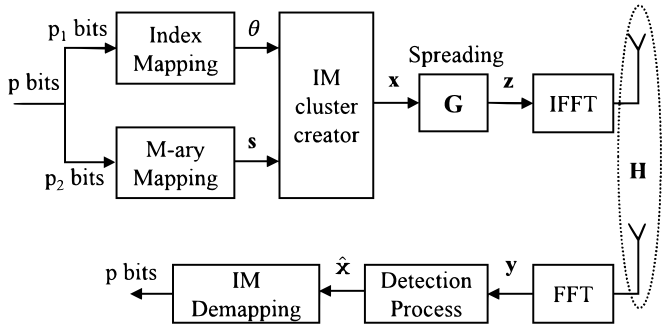

Fig. 1. Block diagram of one cluster of the spread OFDM-IM.

$\theta$ and $\mathbf{s}$ are passed through the IM cluster creator to creat the data symbol vector $\mathbf{x}=\left[x_{1}, \ldots, x_{N}\right]^{T}$, where $x_{i}=0$ for $i \notin \theta$ and $x_{i_{k}}=s_{k}$ for $i_{k} \in \theta$. Unlike the classical scheme, $\mathbf{x}$ is multiplied by the precoding matrix $\mathbf{G}$ before entering the inverse fast Fourier transform (IFFT). Notice that $\mathbf{G}$ is a square matrix with the size of $N \times N$, which can be properly designed to spread both $\theta$ and $\mathbf{s}$, and then compress them into $N$ sub-carriers, harvesting significant diversity gains. We will thoroughly present the design of various precoding matrices afterwards. The precoded vector (denoted by $\mathbf{z}=\mathbf{G x}$ ) is transmitted to the receiver over $N$ flat Rayleigh fading channels. Denote by $\mathbf{H}=\operatorname{diag}\left\{h_{1}, \ldots, h_{N}\right\}$ the frequencydomain channel matrix whose elements $h_{i}$ are independently and identically distributed and $h_{i} \sim \mathcal{C N}\left(0, \sigma^{2}\right)$. Accordingly, the received signal in frequency domain is expressed by

$$
\mathbf{y}=\mathbf{H G x}+\mathbf{n},
$$

where $\mathbf{n}=\left[n_{1}, \ldots, n_{N}\right]^{T}$ is the noise vector with $n_{i} \sim$ $\mathcal{C N}\left(0, N_{0}\right)$. Thus, the signal-to-noise ratio (SNR) can be given as $\bar{\gamma}=\sigma^{2} / N_{0}$. Besides, the data rate (defined as bits per sub-carrier) is given by $R=\left(p_{1}+p_{2}\right) / N$ with $p_{1}=\left\lfloor\log _{2} C(N, K)\right\rfloor$ and $p_{2}=K \log _{2} M$.

Activating all sub-carriers for transmission, S-OFDM-IM suffers from higher complexity of the IFFT process compared to classical OFDM-IM. However, the proposed scheme still inherits benefits of OFDM-IM such as carrying $p_{1}$ index bits without additional power or bandwidth and the trade-off between reliability and spectral efficiency by adjusting $K$.

The receiver can employ the optimal ML detector to estimate the transmitted signal $\hat{\mathbf{x}}$, according to

$$
\hat{\mathbf{x}}=\arg \min _{\mathbf{x}}\|\mathbf{y}-\mathbf{H G} \mathbf{x}\|^{2} .
$$

Utilizing $\hat{\mathbf{x}}$, active indices $\hat{\theta}$ and data symbols $\hat{\mathbf{s}}$ will be recovered, which are then used to demap $p$ bits. Although the ML can achieve the optimal performance, its complexity exponentially grows with $M$ for given $C=2^{p_{1}}$, as $\sim \mathcal{O}\left(C M^{K}\right)$, which is impractical when $K$ and $M$ increase.

\section{B. Spreading Matrices}

We consider two well-known spreading matrices namely Walsh-Hadamard (WH) and Zadoff-Chu (ZC). While the ZC matrix is rarely mentioned in the spread OFDM, the $\mathrm{WH}$ is highly popular [22], which is recursively determined by

$$
\mathbf{G}_{1}=\frac{1}{\sqrt{2}}\left[\begin{array}{cc}
1 & 1 \\
1 & -1
\end{array}\right], \quad \mathbf{G}_{k}=\mathbf{G}_{k-1} \otimes \mathbf{G}_{1} .
$$


This reveals that the size of the WH has to be $N=2^{k}$. Due to all elements being real-valued, this matrix is specifically appropriate for low-complexity implementations. However, the error performance provided by the $\mathrm{WH}$ is less attractive than the $\mathrm{ZC}$ with complex-valued entries. Particularly, to construct the $\mathrm{ZC}$, we resort to the root $\mathrm{ZC}$ sequence as follow

$$
c_{n}=\left\{\begin{array}{ll}
e^{-\frac{j 2 \pi m}{N}\left(\frac{n^{2}}{2}+q n\right)} & \text { for even } N \\
e^{-\frac{j 2 \pi m}{N}\left[\frac{n(n+1)}{2}+q n\right]} & \text { for odd } N
\end{array},\right.
$$

where $q$ is any integer, $m$ is any integer relatively prime to $N$, and $n=1,2, \ldots, N$. The ZC matrix considers $\mathbf{f}_{1}=$ $\left[c_{1}, \ldots, c_{N}\right]^{T}$ as its first column, while its other columns are given as cyclically shifted versions of $\mathbf{f}_{1}$. For example, when $N=4, m=1$ and $q=0$, from (4) we obtain $\mathbf{f}_{1}=[a,-1, a, 1]^{T}$, where $a=(\sqrt{2}+j \sqrt{2}) / 2$. This leads to an $4 \times 4 \mathrm{ZC}$ matrix as follows

$$
\mathbf{G}=\frac{1}{2}\left[\begin{array}{cccc}
a & 1 & a & -1 \\
-1 & a & 1 & a \\
a & -1 & a & 1 \\
1 & a & -1 & a
\end{array}\right]
$$

where note that the factor of $1 / \sqrt{N}$ is to obtain $\|\mathbf{G}\|^{2}=1$.

Apart from being square and invertible, both the $\mathrm{WH}$ and the ZC have two other crucial features [22] as follows: (i) Their entries have the same magnitude. As a result, each non-zero data symbol is equally spread over $N$ sub-carriers, which can allow S-OFDM-IM to harvest a significant diversity gain over existing OFDM-IM schemes; (ii) The two matrices are orthogonal, i.e., $\mathbf{G}^{-1}=\mathbf{G}^{H}$, which aims to enable free-interference transmission and make Euclidean distances between any pairs of $\mathbf{x}$ unchanged before and after spreading. More importantly, the orthogonality of $\mathbf{G}$ also enables a variety of low-complexity detectors as shown in Section IV.

Although the WH and the ZC can help S-OFDM-IM remarkably improve the error performance of OFDM-IM systems, its achievable diversity order is not always maximized for various $N, K$ and $M$. To address this, in the next section, we provide the BEP analysis, followed by the opportunity to use rotated versions of the $\mathrm{WH}$ and the $\mathrm{ZC}$, which can maximize the diversity gain achieved by S-OFDM-IM.

\section{Performance Analysis}

The pairwise error probability (PEP) is evaluated to derive the upper bound on the BEP of S-OFDM-IM. Particularly, the diversity gain and the coding gain are also analyzed, leading to an opportunity of using rotated versions of original $\mathrm{WH}$ and $\mathrm{ZC}$ matrices to further reduce the BEP.

\section{A. BEP Performance Analysis}

The PEP of deciding the signal vector $\hat{\mathbf{x}}$ given that $\mathbf{x}$ is transmitted, conditioned on the channel $\mathbf{H}$, is given by

$$
P(\mathbf{x} \rightarrow \hat{\mathbf{x}} \mid \mathbf{H})=Q\left(\sqrt{\frac{\|\mathbf{H G}(\mathbf{x}-\hat{\mathbf{x}})\|^{2}}{2 N_{0}}}\right),
$$

where $Q($.$) denotes the Gaussian tail probability [26]. Let$ $\Omega=\|\mathbf{H G}(\mathbf{x}-\hat{\mathbf{x}})\|^{2}$, which can be rewritten as

$$
\Omega=\sum_{i=1}^{N} \eta_{i}\left|h_{i}\right|^{2}
$$

where $\eta_{i}=\left|\mathbf{g}_{i}(\mathbf{x}-\hat{\mathbf{x}})\right|^{2}$ with $\mathbf{g}_{i}$ is the $i$-th row of $\mathbf{G}$. Thus, we can represent (6), using the alternative form of Q-function [26], as

$$
P(\mathbf{x} \rightarrow \hat{\mathbf{x}} \mid \mathbf{H})=\frac{1}{\pi} \int_{0}^{\pi / 2} \exp \left(-\frac{\Omega}{4 N_{0} \sin ^{2} \phi}\right) d \phi
$$

By averaging (8) with respect to $\Omega$, the unconditional PEP is obtained by $P(\mathbf{x} \rightarrow \hat{\mathbf{x}})=\frac{1}{\pi} \int_{0}^{\pi / 2} \mathcal{M}_{\Omega}\left(-\frac{1}{4 N_{0} \sin ^{2} \phi}\right) d \phi$, where $\mathcal{M}_{\Omega}(t)$ is the moment generating function (MGF) of $\Omega$. Due to the channel model, the MGF of $\Lambda_{i}=\eta_{i}\left|h_{i}\right|^{2}$ in (7) is $\mathcal{M}_{\Lambda_{i}}(t)=\left(1-\eta_{i} \sigma^{2} t\right)^{-1}$, which leads to $\mathcal{M}_{\Omega}(t)=$ $\prod_{i=1}^{N}\left(1-\eta_{i} \sigma^{2} t\right)^{-1}$, thus we obtain

$$
P(\mathbf{x} \rightarrow \hat{\mathbf{x}})=\frac{1}{\pi} \int_{0}^{\pi / 2} \prod_{i=1}^{N}\left(\frac{\sin ^{2} \phi}{\sin ^{2} \phi+\frac{\eta_{i} \sigma^{2}}{4 N_{0}}}\right) d \phi .
$$

For a given spreading matrix $\mathbf{G}$, let us define the set $\mathcal{G}_{\mathbf{x}, \hat{\mathbf{x}}}=$ $\left\{i \mid \eta_{i} \neq 0\right\}$ and denote its cardinality $\Gamma_{\mathbf{x}, \hat{\mathbf{x}}}=\left|\mathcal{G}_{\mathbf{x}, \hat{\mathbf{x}}}\right|$. Because of the fact that $0 \leq \sin ^{2} \phi \leq 1$, we can approximate $\sin ^{2} \phi\left(\sin ^{2} \phi+\eta_{i} \sigma^{2} / 4 N_{0}\right)^{-1} \leq\left(1+\eta_{i} \sigma^{2} / 4 N_{0}\right)^{-1} \approx$ $4 / \eta_{i} \bar{\gamma}$ at high SNRs, for $i \in \mathcal{G}_{\mathbf{x}, \hat{\mathbf{x}}}$. Thus, the integrand in (9) can be approximated by

$$
P(\mathbf{x} \rightarrow \hat{\mathbf{x}}) \approx \frac{(\bar{\gamma} / 4)^{-\Gamma_{\mathbf{x}, \hat{\mathbf{x}}}}}{2 \prod_{i \in \mathcal{G}_{\mathbf{x}, \hat{\mathbf{x}}}} \eta_{i}} .
$$

It is seen from (10) that the diversity order of the PEP is $\Gamma_{\mathbf{x}, \hat{\mathbf{x}}}$, or equivalently, the number of non-zero elements of the precoded vector $\mathbf{G}(\mathbf{x}-\hat{\mathbf{x}})$, which is strongly influenced by the choice of $\mathbf{G}$. Using (10), the diversity and coding gains achieved by S-OFDM-IM are respectively computed as

$$
\begin{gathered}
G_{d}=\min _{\mathbf{x} \neq \hat{\mathbf{x}}} \Gamma_{\mathbf{x}, \hat{\mathbf{x}}}, \\
G_{c}=\min _{\mathbf{x} \neq \hat{\mathbf{x}}, \Gamma_{\mathbf{x}, \hat{\mathbf{x}}}=G_{d}}\left(\prod_{i \in \mathcal{G}_{\mathbf{x}, \hat{\mathbf{x}}}} \eta_{i}\right)^{\frac{1}{G_{d}}} .
\end{gathered}
$$

After the evaluation of $P(\mathbf{x} \rightarrow \hat{\mathbf{x}})$, using the union bound theory, we can attain the upper bound on the BEP as follows

$$
P_{b} \leq \frac{1}{p C M^{K}} \sum_{\mathbf{x}} \sum_{\hat{\mathbf{x}}} \frac{w(\mathbf{x}, \hat{\mathbf{x}})(\bar{\gamma} / 4)^{-\Gamma_{\mathbf{x}, \hat{\mathbf{x}}}}}{2 \prod_{i \in \mathcal{G}_{\mathbf{x}, \hat{\mathbf{x}}}} \eta_{i}},
$$

where $w(\mathbf{x}, \hat{\mathbf{x}})$ is number of different bits between $\mathbf{x}$ and $\hat{\mathbf{x}}$.

Remark 1: As shown in (11) and (12), for given system parameters $N, K$ and $M$, it is desirable to design a spreading matrix $\mathbf{G}$ to maximize both the diversity gain and the coding gain of S-OFDM-IM. For simplicity, employing the WH and $\mathrm{ZC}$ matrices to the proposed scheme, we expect to remarkably enhance the BEP performance of OFDM-IM schemes. For example, when $N=4, K=1$ and $M=4$, S-OFDM-IM schemes using the $\mathrm{WH}$ and the $\mathrm{ZC}$ achieve the same diversity and coding gains as $G_{d}=2$ and $G_{c}=1$. Hence, the diversity 
TABLE I

DIVERSITY AND CODING GAIN $\left(G_{d}-G_{c}\right)$ COMPARISON AMONG FOUR SPREADING MATRICES, WITH VARIOUS S-OFDM-IM CONFIGURATIONS

\begin{tabular}{|c|c|c|c|c|}
\hline$(N, K, M)$ & $\mathrm{WH}$ & $\mathrm{ZC}$ & roWH & roZC \\
\hline \hline$(4,1,4)$ & $2-1$ & $2-1$ & $4-0.1913$ & $4-0.2973$ \\
\hline$(4,2,4)$ & $1-4$ & $2-1.4142$ & $4-0.1913$ & $4-0.2973$ \\
\hline$(4,2,16)$ & $1-0.08$ & $2-0.0283$ & $3-0.0023$ & $3-0.0532$ \\
\hline$(8,2,4)$ & $2-2$ & $2-2$ & $8-0.0373$ & $8-0.1363$ \\
\hline
\end{tabular}

gain of S-OFDM-IM is twice larger than that of OFDM-IM. However, increasing diversity gain may lead to the degradation in the constrained capacity of OFDM-IM as analyzed in [9].

Remark 2: In spite of having the same diversity and coding gains, the $\mathrm{WH}$ and the $\mathrm{ZC}$ may provide different performances. This is because two matrices can result in distinct kissing numbers, which are defined as the total number of pairs $(\mathbf{x}, \hat{\mathbf{x}})$ that have the minimum diversity and coding gains, i.e., $G_{d}$ and $G_{c}$. Particularly, the one with a smaller kissing number is preferred to reduce the BEP. In the above example, kissing numbers of the $\mathrm{WH}$ and the $\mathrm{ZC}$ are 24 and 8, respectively. Thus, the $\mathrm{ZC}$ is expected to have a better BEP than the WH.

Remark 3: The above example also reveals that the $\mathrm{WH}$ and the $\mathrm{ZC}$ do not always provide a maximum diversity gain, i.e., $N$, for S-OFDM-IM. In other words, using the two matrices, there may exist pairs of $(\mathbf{x}, \hat{\mathbf{x}})$ satisfying $\Gamma_{\mathbf{x}, \hat{\mathbf{x}}}<N$. Interestingly, this is caused by the fact that all of rows of either the $\mathrm{WH}$ or the $\mathrm{ZC}$ have at least two elements being the same. To illustrate this, let us consider an instance as follows. For $N=4$, the $\mathrm{ZC}$ matrix in (5) has the first row of $\mathbf{g}_{1}=\left[\begin{array}{llll}\bar{a} & 0.5 & \bar{a} & -0.5\end{array}\right]$, where $\bar{a}=a / 2$. Meanwhile, for any $K<N$, there always exists $(\mathbf{x}, \hat{\mathbf{x}})$ with $\mathbf{x}-\hat{\mathbf{x}}=\left[\begin{array}{cccc}s & 0 & -s & 0\end{array}\right]^{T}$, where $s \in \mathcal{S}$. Thus, we obtain $\left|\mathbf{g}_{1}(\mathbf{x}-\hat{\mathbf{x}})\right|^{2}=|\bar{a} s+\bar{a}(-s)|^{2}=0$, leading to $\Gamma_{\mathbf{x}, \hat{\mathbf{x}}}<N$.

\section{B. Opportunity of Angle Rotated Spreading Matrices}

As a result of Remark 3, we further investigate to rotate each column by different angles to make each row unequal. This simple method can increase $\Gamma_{\mathbf{x}, \hat{\mathbf{x}}}$, or equivalently, enlarge the diversity gain of S-OFDM-IM over the original WH and $\mathrm{ZC}$ matrices. In particular, we propose to use rotated spreading matrices for S-OFDM-IM, which are determined by rotating every column of original matrices $\mathbf{u}_{i}$ by distinct angles,

$$
\overline{\mathbf{u}}_{i}=\mathbf{u}_{i} \times \exp \left[\frac{j 2 \pi(i-1)}{M N}\right],
$$

where $\overline{\mathbf{u}}_{i}$ is the $i$-th column of rotated matrices for $i=$ $1, \ldots, N$. The above selected angles can be seen in [22], which proposes rotated transforms of the $\mathrm{WH}$ and Fourier matrices for S-OFDM. However, it is worth noting that such the rotation method applied to the ZC has not yet been proposed in the literature, even for the classical OFDM. This work first sheds light on the potential of using the rotated $\mathrm{ZC}$ matrix in the spread OFDM-IM over other candidates.

More precisely, Table I compares performance gains provided by four spreading matrices, namely $\mathrm{WH}, \mathrm{ZC}$ and their rotated versions (denoted as roWH and roZC), under various

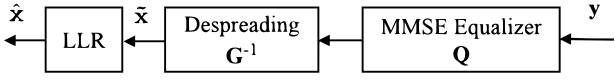

Fig. 2. The proposed MMSE-LLR detector.

configurations of $(N, K, M)$ with the $M$-ary QAM modulation. As shown in Table I, rotated matrices can offer the maximum diversity gain, i.e., $N$, thus achieve the superior performance over original ones. Especially, the proposed roZC always provides the best performance.

\section{LOW-COMPLEXITy Detectors}

To implement S-OFDM-IM in practice, we now propose three low-complexity detectors that achieve different levels of complexity and error performance. This leads to a balanced trade-off between complexity and performance when selecting the detector type at the receiver. The complexity analysis and comparison among proposed detectors are also presented.

\section{A. MMSE-LLR Detector}

The MMSE-LLR is based on the minimum mean square error (MMSE) equalizer and the log-likelihood (LLR) method, as illustrated in Fig. 2. Particularly, the received signal vector $\mathbf{y}$ is first multiplied by the MMSE equalization matrix which is denoted as $\mathbf{Q}=\operatorname{diag}\left\{q_{1}, q_{2}, \ldots, q_{N}\right\}$, where

$$
q_{i}=\frac{h_{i}^{*}}{\left|h_{i}\right|^{2}+\bar{\gamma}^{-1}} .
$$

The output of the channel equalizer is then multiplied by the despreading matrix $\mathbf{G}^{-1}\left(=\mathbf{G}^{H}\right)$ to extract the received data symbol vector as follows

$$
\tilde{\mathbf{x}}=\mathbf{G}^{H} \mathbf{Q} \mathbf{y} .
$$

It is noteworthy that there are no inverse matrix operators required in (16) due to the orthogonality of the $\mathrm{ZF}, \mathrm{WH}$ matrices and their rotated versions. As a result, a substantial reduction in the computational complexity can be achieved.

After despreading, the LLR method is used to estimate the data symbol vector $\hat{\mathbf{x}}$ through calculating the following LLR for each sub-carrier (see [19] for more details)

$$
\lambda_{i}=\left|\tilde{x}_{i}\right|^{2}-\left|\tilde{x}_{i}-\mathcal{D}\left(\tilde{x}_{i}\right)\right|^{2}, \text { for } i=1, \ldots, N
$$

where $\tilde{x}_{i}$ is the $i$-th element of $\tilde{\mathbf{x}}$, and $\mathcal{D}(x)$ is the digital demodulator function that returns the $M$-ary symbol which is the most likely to $x$. Finally, based on $K$ largest LLRs $\lambda_{i}$ from (17), we can recover $\hat{\mathbf{x}}$, or equivalently, active indices $\hat{\theta}$ and corresponding $K$ data symbols $\hat{\mathrm{s}}$. The MMSE-LLR can be described in Algorithm 1.

Notice from [27] that despite achieving a remarkably lower complexity compared to the ML, there is still a notable performance gap between the MMSE-LLR and the ML. 


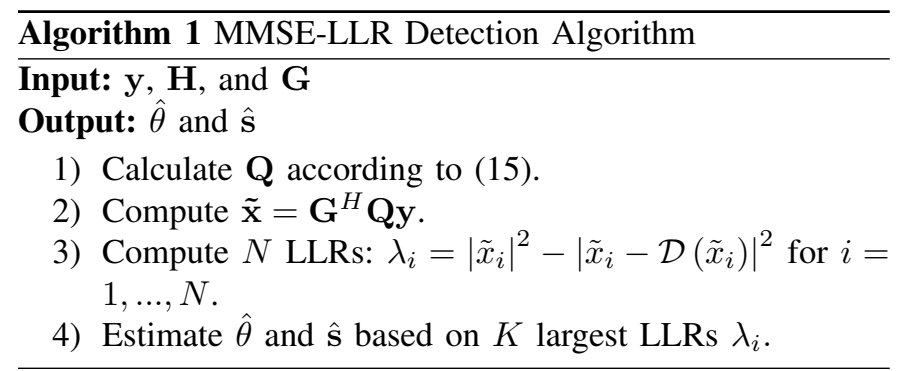

\section{B. IP-MMSE Detector}

We now propose a novel detector termed as index pattern MMSE (IP-MMSE), which can attain the near-optimal performance at much lower complexity than the ML. Of course, the IP-MMSE is more complicated than the MMSE-LLR.

Particularly, denote by $\mathcal{F}=\left\{\theta_{1}, \ldots, \theta_{C}\right\}$ the set of all index patterns used in S-OFDM-IM for given $N$ and $K$. In the IPMMSE, for each index pattern $\theta_{c}$ in $\mathcal{F}$, we define $\mathbf{H}_{c}$ as the sub-matrix that contains $K$ columns of $\overline{\mathbf{H}}$ whose indices are in $\theta_{c}$, where $\overline{\mathbf{H}}=\mathbf{H G}$ is an $N \times N$ matrix. The MMSE equalization matrix for the channel pattern $\mathbf{H}_{c}$ is given by

$$
\mathbf{Q}_{c}=\left(\mathbf{H}_{c}^{H} \mathbf{H}_{c}+\bar{\gamma}^{-1} \mathbf{I}\right)^{-1} \mathbf{H}_{c}^{H},
$$

where $\mathbf{I}$ is the $K \times K$ identity matrix. Then, the corresponding $K$ data symbols is estimated using the MMSE detector, as

$$
\hat{\mathbf{s}}_{c}=\mathcal{D}\left(\mathbf{Q}_{c} \mathbf{y}\right) .
$$

Next, we calculate the distance between the received signal $\mathbf{y}$ and the estimated non-zero data symbols $\hat{\mathbf{s}}_{c}$, as follows $w_{c}=$ $\left\|\mathbf{y}-\mathbf{H}_{c} \hat{\mathbf{s}}_{c}\right\|^{2}$. Finally, the index pattern $\hat{\theta}$ and $K$ complex $M$-ary sysmbols $\hat{\mathbf{s}}$ are respectively recovered by

$$
\hat{\theta}=\theta_{\hat{c}}, \hat{\mathbf{s}}=\hat{\mathbf{s}}_{\hat{c}}, \text { where } \hat{c}=\arg \min _{c=1, \ldots, C} w_{c} .
$$

The IP-MMSE detector can be summarized in Algorithm 2.

Notice that compared to another similar approach, named as ordered block-MMSE (OS-MMSE) [28], IP-MMSE does not involve an ordering algorithm of possible index patterns which needs a termination threshold based on sphere detection. Thus, IP-MMSE can offer better performance than OS-MMSE at a comparable complexity. Moreover, in contrast to OS-MMSE, our detector has the complexity not depending on the SNR.

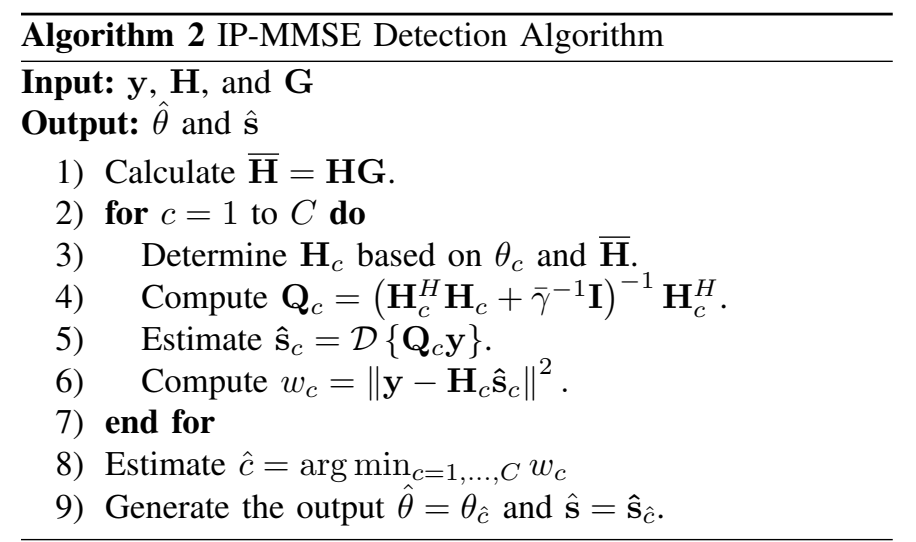

\section{Enhanced IP-MMSE Detector}

It should be noted that the IP-MMSE encounters an $K \times K$ matrix inverse at each for loop, which can result in a burden of complexity, especially when $K$ increases. To tackle this issue, we propose an enhanced IP-MMSE (EIP-MMSE) detector without encountering any matrix inverse computations.

In particular, for each index pattern $\theta_{c}$, let us define the sub-matrix $\mathbf{G}_{c}$ as $K$ columns of $\mathbf{G}$ whose indices are in $\theta_{c}$. Unlike the IP-MMSE detector, the EIP-MMSE calculates the equalization matrix for each index pattern simply as follows

$$
\mathbf{Q}_{c}=\mathbf{G}_{c}^{H} \mathbf{Q}
$$

where $\mathbf{Q}$ is given in (15). After obtaining $\mathbf{Q}_{c}$, the rest of the this detection algorithm is similar to that of the IP-MMSE, except for computing $w_{c}=\left\|\mathbf{y}-\mathbf{H G}_{c} \hat{\mathbf{s}}_{c}\right\|^{2}$. The EIP-MMSE detector can be demonstrated in Algorithm 3.

The difference between IP-MMSE and EIP-MMSE is in step 4, where unlike the former, the latter does not require any inverse matrix calculations in this step and therefore achieves a reduced complexity. However, this can result in the performance loss of EIP-MMSE compared to IP-MMSE.

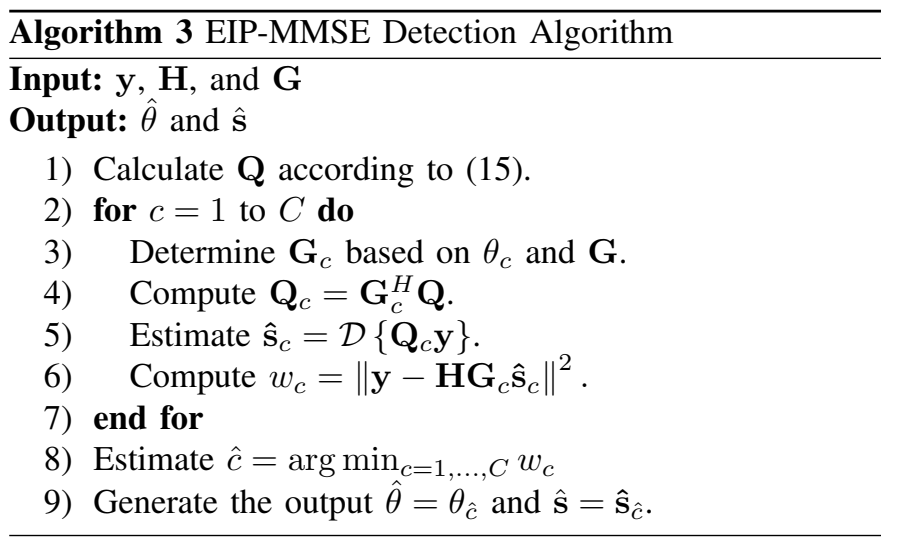

Remark 4: By combining the IM concept and orthogonal precoding matrices, S-OFDM-IM enables a number of lowcomplexity, near-optimal detectors as presented above. However, such the benefit is not available in S-OFDM since this scheme carries $N M$-ary data symbols only, and without any index symbol. This will also be validated in simulation results. In addition, some recent detectors based on the sequential Monte Carlo method [29] and the sphere detection [30] can be developed for S-OFDM-IM to attain near-ML performance.

\section{Complexity Analysis and Comparison}

The computational complexities of proposed detectors are evaluated in terms of the number of floating point operations (flops) per sub-carrier. It is assumed that a flop can be either a real square root, a real division, a real multiplication, or a real summation. For instance, a complex multiplication and a complex summation are counted as 6 flops and 2 flops, respectively. We also assume the spreading matrix $\mathbf{G}$ to be complex-valued for calculations.

Based on above assumptions, the complexities of three proposed detectors and the ML are calculated in Table II. As shown in this table, unlike the ML, the complexities of our 
TABLE II

DETECTION COMPLEXITY COMPARISONS IN TERMS OF NUMBER OF FLOPS PER SUB-CARRIER

\begin{tabular}{|c|c|c|}
\hline Detector & Complexity (flops/sub-carrier) & Order of complexity \\
\hline \hline ML & $(8 N+9) C M^{K}$ & $\mathcal{O}\left(N C M^{K}\right)$ \\
\hline MMSE-LLR & $8 N+19$ & $\mathcal{O}(N)$ \\
\hline IP-MMSE & $C\left(8 K^{2}+16 K+3\right)+6 N+6 C\left(K^{3}+K^{2}\right) / N$ & $\mathcal{O}\left(C K^{2}\right)$ \\
\hline EIP-MMSE & $14 C K+9 C+6$ & $\mathcal{O}(C K)$ \\
\hline
\end{tabular}
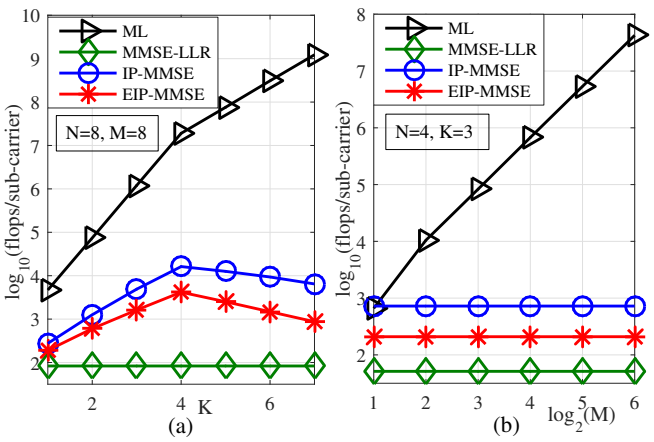

Fig. 3. Computational complexity comparisons between three proposed detectors and the ML when (a) $N=8, M=8, K=1, \ldots, 7$ and (b) $N=4, K=3, M=2,4, \ldots, 64$.

detectors are all independent of $M$. In other words, increasing $M$ does not increase their computational complexities. It also should be noted that the MMSE-LLR provides the lowest complexity, which depends on $N$ only. Among three proposed detectors, the IP-MMSE offers the highest complexity which is mainly caused by the calculation of the matrix inverse at each iteration. By contrast, the EIP-MMSE requires significantly less flops per sub-carrier than the IP-MMSE as expected.

To clearly illustrate above conclusions, we provide Fig. 3 that depicts computational complexities of four mentioned detectors when increasing either $K$ or $M$. This figure once again validates the superiority of proposed detectors in terms of complexity over the ML, especially when $K$ and $M$ gets larger. For example, when $(N, K, M)=(4,3,16)$, as shown in Fig. 3(b), the ML requires 671744 flops which is substantially larger that of the proposed MMSE-LLR, IP-MMSE and EIPMMSE with 51, 732 and 210 flops, respectively. Also, it is shown from Fig. 3(a) that when $K \simeq N / 2$, complexities of both the IP-MMSE and the EIP-MMSE are highest. This can be explained that the number of index patterns, i.e., $C$ becomes much larger when $K$ tends to $N / 2$.

In terms of reliability, the IP-MMSE attains the best performance which is close to the ML, while the EIP-MMSE performs much better than MMSE-LLR, as verified in Section $\mathrm{V}$. Consequently, a balanced trade-off between complexity and reliability can be achieved just by selecting detection types.

\section{Simulation RESUlTS}

We now demonstrate the BEP of S-OFDM-IM with various spreading matrices and detector types through simulation results. To show the superiority of the proposed scheme, we select IM-OFDM-SS [18], CI-OFDM-IM [15], classical OFDM-IM [3], OFDM and S-OFDM [22] as benchmark
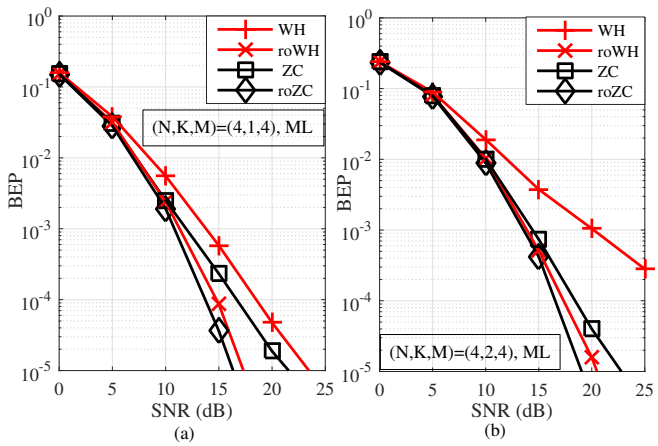

Fig. 4. BEP performance of S-OFDM-IM using four spreading matrices, i.e., WH, ZC, roWH and roZC, when (a) $(N, K, M)=(4,1,4)$ and (b) $(N, K, M)=(4,2,4)$, and ML detector is used.

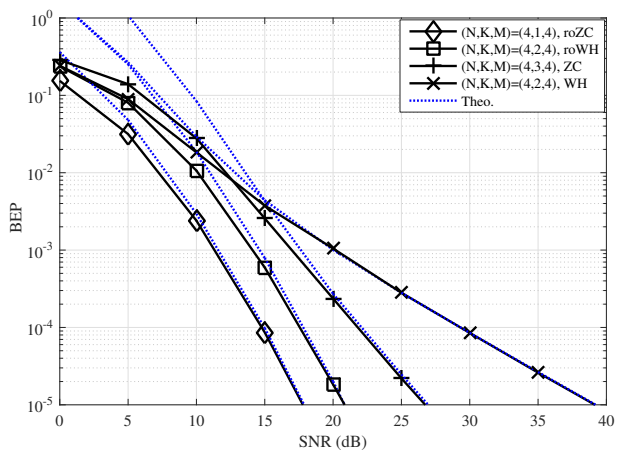

Fig. 5. Comparison between theoretical upper bounds on the BEP of SOFDM-IM and simulation results when various configurations of $(N, K, M)$ and spreading matrices are employed.

schemes. For simplicity, configurations of S-OFDM-IM, CIOFDM-IM, OFDM-IM are referred to as $(N, K, M)$, while that of OFDM-IM-SS and S-OFDM is $(N, M)$, where $N$ and $K$ are the number of sub-carriers and active sub-carrier per cluster, respectively, and $M$ is the modulation size. For all schemes, the PSK modulation is used when $M \leq 8$, whereas we employ the QAM modulation for $M>8$.

Fig. 4 depicts the BEPs of two S-OFDM-IM schemes with four spreading matrices, namely $\mathrm{WH}, \mathrm{ZC}$, roWH and roZC, and the ML detection. As seen from Fig. 4, rotated matrices obviously provide the superior performance over original ones as analyzed in Section III. For example, at the BEP of $10^{-4}$ in Fig. 4(a), the roWH and roZC achieve SNR gains of $3 \mathrm{~dB}$ and $4 \mathrm{~dB}$, respectively, over their original versions. Furthermore, the $\mathrm{ZC}$ and its rotated version are more preferred than the $\mathrm{WH}$ counterparts, which confirms Remark 2. Specifically, the roZC always offers the best performance among four choices. Thus, hereinafter, we will employ the roZC in S-OFDM-IM as well 


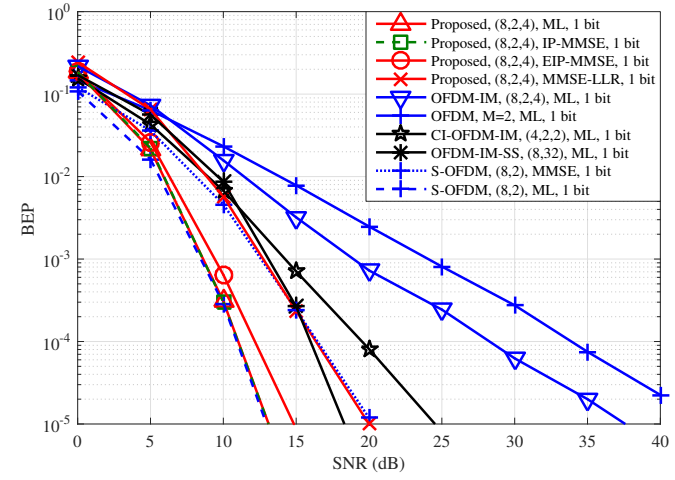

Fig. 6. BEP comparison between S-OFDM-IM and reference schemes at 1 bit per sub-carrier. Reference schemes uses the ML, while the proposed scheme employs the ML and the proposed MMSE-LLR, IP-MMSE, and EIP-MMSE.

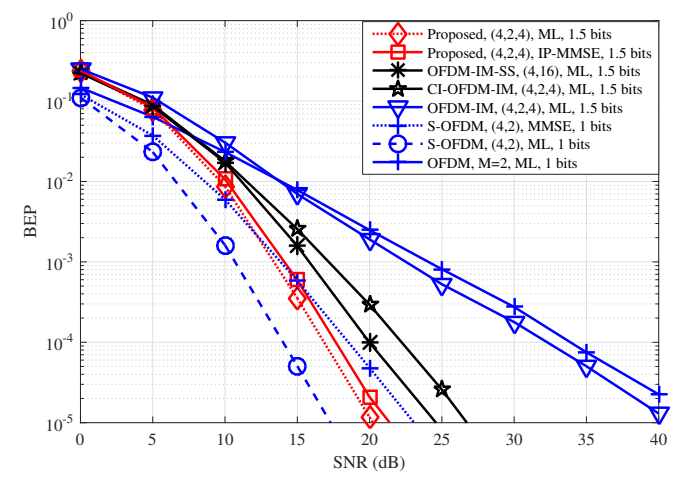

Fig. 7. BEP comparison between S-OFDM-IM and reference schemes at the data rate of 1.5 bits per sub-carrier. Reference schemes uses the ML, while the proposed scheme employs either the ML or the proposed IP-MMSE.

as S-OFDM for comparisons.

In Fig. 5, we compare the theoretical bounds on the BEP with simulation results, under different $(N, K, M)$ and spreading matrices. As we can see, the theoretical bounds are tight in all cases, especially at increasing SNRs and BEPs of less than $10^{-3}$. Hence, the bound can be a useful tool to evaluate the BEP of the proposed scheme at high SNRs.

Fig. 6 compares the BEPs between S-OFDM-IM and its benchmarks, at 1 bit per sub-carrier. The ML is used for all benchmark schemes, while S-OFDM-IM employs either the ML or three proposed detectors. As seen from Fig 6, despite employing low-complexity detectors such as IP-MMSE and EIP-MMSE, the proposed scheme still significantly outperforms all benchmarks with the ML, except for S-OFDM. For instance, at the BEP of $10^{-3}$, S-OFDM-IM with either the ML or the IP-MMSE provides considerable SNR gains of 5, 6, 10 and $15 \mathrm{~dB}$ over IM-OFDM-SS, CI-OFDM-IM, classical OFDM-IM and OFDM, respectively. This is because our scheme achieves diversity order of 8 as shown in Table I, while benchmarks such as OFDM-IM or OFDM offers the unit diversity order only. Compared to S-OFDM, S-OFDM-IM has the similar BEP when the ML is used. However, when MMSEbased detectors are used, S-OFDM-IM notably outperforms S-OFDM. The reason is that our detectors, for example, IPMMSE exploits all possible MMSE matrices according to

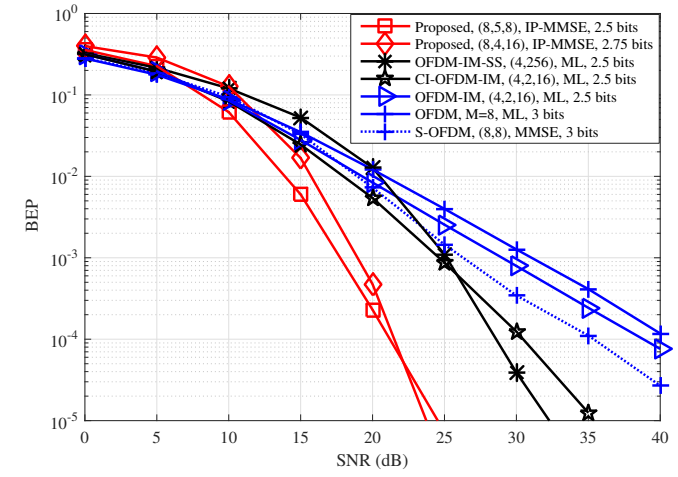

Fig. 8. BEP comparison between S-OFDM-IM and reference schemes at the data rate of 2.5 bits per sub-carrier. Reference schemes uses the ML, while the proposed scheme employs the IP-MMSE.

index patterns to simultaneously detect both the index and $M$ ary symbols, which makes S-OFDM-IM retain the diversity gain better than that of S-OFDM which has no IM process. This clearly confirms the benefit of the proposed scheme in terms of receiver design over S-OFDM presented in Remark 4. In addition, the IP-MMSE can achieve a near-optimal BEP as the ML, while the EIP-MMSE and the MMSE-LLR suffers slight and remarkable performance losses, respectively. Thus, we mainly use the IP-MMSE for S-OFDM-IM, hereinafter, to compare with other systems at higher rates.

Fig. 7 illustrates the BEP comparison between S-OFDMIM and benchmark schemes at 1.5 bits per sub-carrier. Our proposed S-OFDM-IM employs either the ML or the IPMMSE detector, while others use the ML. Since S-OFDM does not work at 1.5 bits per sub-carrier, we provide the curve of S-OFDM with $(N, M)=(4,2)$. It can be found from this figure that there is a slight performance loss caused by the IPMMSE in comparison with the ML. However, the proposed IPMMSE once again performs better than benchmark schemes employing the ML. Especially, applying the MMSE-based detector, S-OFDM-IM with 1.5 bits performs even better than S-OFDM with 1 bit, whereas this can not be achieved when the ML detector is used.

In Fig. 8, we compare S-OFDM-IM with reference schemes at 2.5 bits per sub-carrier. Notice that at higher data rate, the proposed scheme may require larger $K$ and $M$ as shown in Fig. 8, which makes the ML impractical for signal detections. Thus, we only show the BEP of the proposed IP-MMSE for comparisons. Similarly, we include the BEP of S-OFDM with the MMSE only in this figure. Interestingly, as seen from Fig. 8, in spite of just using the IP-MMSE detector, S-OFDMIM still provides a superior performance over the benchmarks with the ML. For example, at the BEP of $10^{-4}$, the proposed scheme of $(8,4,16)$ can attain SNR gains of 5, 7, 17, 19, 14 over IM-OFDM-SS, CI-OFDM-IM, OFDM-IM, OFDM, and S-OFDM, respectively. In addition, our scheme with 2.75 bits has better performance than that with 2.5 bits at high SNRs. This may be due to the different diversity gains between them.

In summary, employing the roZC and proposed detectors, S-OFDM-IM can significantly improve the BEP over current schemes. The proposed system also inherits a range of advan- 
tages from OFDM-IM such as higher energy efficiency and flexibility over classical OFDM. These make the proposed SOFDM-IM more appropriate for critical machine type communications which require very high reliability at low complexity.

\section{CONCLUSIONS}

We proposed a novel S-OFDM-IM scheme to employ various spreading matrices in the OFDM-IM framework in order to enhance the reliability of OFDM-IM. Particularly, adopting WH and ZC spreading matrices, S-OFDM-IM spreads both non-zero data and index symbols to all available sub-carriers, and then compress them into each subcarrier. The proposed scheme was analyzed to benefit from both multipath and index diversities, thus significantly increase the transmit diversity of OFDM-IM. The analyzed BEP provided an insight into impacts of various spreading matrices on achievable diversity and coding gains. Based on this, we discovered potentials of using rotated $\mathrm{WH}$ and $\mathrm{ZC}$ matrices to further improve the performance over their original versions. Especially, these rotated matrices allows S-OFDM-IM to achieve the maximum diversity gain, in which the rotated ZC always exhibits the best performance. We proposed three low-complexity detectors, namely MMSE-LLR, IP-MMSE, and EIP-MMSE, whose complexities are all independent of $M$. Simulation results clearly show that S-OFDM-IM is superior to benchmarks schemes, even when using lower complexity detectors.

\section{REFERENCES}

[1] S. Sasaki, J. Zhu, and G. Marubayashi, "Performance of parallel combinatory spread spectrum multiple access communication systems," in Proc. IEEE Pers., Indoor., Mobile Radio Commun., Sep 1991, pp. 204-208.

[2] P. K. Frenger and N. A. B. Svensson, "Parallel combinatory OFDM signaling," IEEE Trans. Commun., vol. 47, no. 4, pp. 558-567, Apr 1999.

[3] E. Basar, U. Aygolu, E. Panayirci, and H. V. Poor, "Orthogonal frequency division multiplexing with index modulation," IEEE Trans. Signal Process., vol. 61, no. 22, pp. 5536-5549, Nov 2013.

[4] E. Basar, M. Wen, R. Mesleh, M. D. Renzo, Y. Xiao, and H. Haas, "Index modulation techniques for next-generation wireless networks," IEEE Access, vol. 5, pp. 16693-16746, 2017.

[5] X. Cheng, M. Zhang, M. Wen, and L. Yang, "Index modulation for 5G: Striving to do more with less," IEEE Wireless Commun, vol. 25, no. 2, pp. 126-132, April 2018.

[6] R. Fan, Y. J. Yu, and Y. L. Guan, "Generalization of orthogonal frequency division multiplexing with index modulation," IEEE Trans. Wireless Commun., vol. 14, no. 10, pp. 5350-5359, Oct 2015.

[7] J. Crawford and Y. Ko, "Low complexity greedy detection method with generalized multicarrier index keying OFDM," in Proc. IEEE Pers., Indoor., Mobile Radio Commun., Aug 2015, pp. 688-693.

[8] T. V. Luong and Y. Ko, "A tight bound on BER of MCIK-OFDM with greedy detection and imperfect CSI," IEEE Commun. Lett., vol. 21, no. 12 , pp. 2594 - 2597, Dec 2017.

[9] M. Wen, X. Cheng, M. Ma, B. Jiao, and H. V. Poor, "On the achievable rate of OFDM with index modulation," IEEE Trans. Signal Process., vol. 64, no. 8, pp. 1919-1932, April 2016.

[10] Y. Ko, "A tight upper bound on bit error rate of joint OFDM and multicarrier index keying," IEEE Commun. Lett., vol. 18, no. 10, pp. 1763 1766, Oct 2014.

[11] E. Basar, "On multiple-input multiple-output OFDM with index modulation for next generation wireless networks," IEEE Trans. Signal Process. vol. 64, no. 15, pp. 3868-3878, Aug 2016.

[12] T. V. Luong and Y. Ko, "Impact of CSI uncertainty on MCIK-OFDM: tight, closed-form symbol error probability analysis," IEEE Trans. Veh. Technol., vol. 67, no. 2, pp. 1272 - 1279, Feb 2018.

[13] T. Mao, Z. Wang, Q. Wang, S. Chen, and L. Hanzo, "Dual-mode index modulation aided OFDM," IEEE Access, vol. 5, pp. 50-60, 2017.
[14] Q. Li, M. Wen, E. Basar, H. V. Poor, B. Zheng, and F. Chen, "Diversity enhancing multiple-mode OFDM with index modulation," IEEE Trans. Commun., to be published.

[15] E. Basar, "OFDM with index modulation using coordinate interleaving," IEEE Wireless Commun. Lett., vol. 4, no. 4, pp. 381-384, Aug 2015.

[16] H. Zhang, L. L. Yang, and L. Hanzo, "Compressed sensing improves the performance of subcarrier index-modulation-assisted OFDM," IEEE Access, vol. 4, pp. 7859-7873, 2016.

[17] G. Kaddoum, M. F. A. Ahmed, and Y. Nijsure, "Code index modulation: A high data rate and energy efficient communication system," IEEE Commun. Lett., vol. 19, no. 2, pp. 175-178, Feb 2015.

[18] Q. Li, M. Wen, E. Basar, and F. Chen, "Index modulated OFDM spread spectrum," IEEE Trans. Wireless Commun., vol. 17, no. 4, pp. 23602374,2018

[19] J. Choi, "Coded OFDM-IM with transmit diversity," IEEE Trans. Commun., vol. 65, no. 7, pp. 3164-3171, July 2017.

[20] T. V. Luong, Y. Ko, and J. Choi, "Repeated MCIK-OFDM with enhanced transmit diversity under CSI uncertainty," IEEE Trans. Wireless Commun., vol. 17, no. 6, pp. 4079-4088, June 2018.

[21] Z. Liu, Y. Xin, and G. B. Giannakis, "Linear constellation precoding for OFDM with maximum multipath diversity and coding gains," IEEE Trans. Commun., vol. 51, no. 3, pp. 416-427, March 2003.

[22] A. Bury, J. Egle, and J. Lindner, "Diversity comparison of spreading transforms for multicarrier spread spectrum transmission," IEEE Trans. Commun., vol. 51, no. 5, pp. 774-781, May 2003.

[23] M. Wen, B. Ye, E. Basar, Q. Li, and F. Ji, "Enhanced orthogonal frequency division multiplexing with index modulation," IEEE Trans. Wireless Commun., vol. 16, no. 7, pp. 4786 - 4801, July 2017.

[24] J. C. Inacio, B. F. Uchoa-Filho, and D. L. Ruyet, "Exploiting signal space diversity in OFDM with grouped subcarriers: Going beyond subcarrier index modulation," IEEE Wireless Commun. Lett., to be published.

[25] S. Gao, M. Zhang, and X. Cheng, "Precoded index modulation for multiinput multi-output OFDM," IEEE Trans. Wireless Commun., vol. 17, no. 1, pp. 17-28, Jan 2018.

[26] M. K. Simon and M. S. Alouini, Digital Communication over Fading Channels. 2nd edition. John \& Wiley, 2005.

[27] T. V. Luong and Y. Ko, "Precoding for spread OFDM IM," in Proc. IEEE 87th VTC-Spring 2018.

[28] Y. Xiao, Z. Yang, L. Dan, P. Yang, L. Yin, and W. Xiang, "Lowcomplexity signal detection for generalized spatial modulation," IEEE Commun. Lett., vol. 18, no. 3, pp. 403-406, March 2014.

[29] B. Zheng, M. Wen, E. Basar, and F. Chen, "Multiple-input multipleoutput OFDM with index modulation: Low-complexity detector design," IEEE Trans. Signal Process., vol. 65, no. 11, pp. 2758-2772, June 2017.

[30] B. Zheng, M. Wen, F. Chen, N. Huang, F. Ji, and H. Yu, "The K-best sphere decoding for soft detection of generalized spatial modulation," IEEE Trans. Commun., vol. 65, no. 11, pp. 4803-4816, Nov 2017.

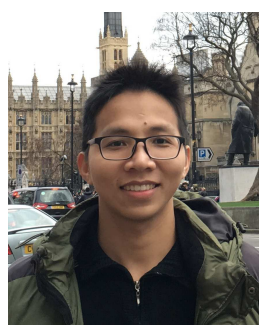

Thien Van Luong received the B.S degree in electrical engineering from Hanoi University of Science and Technology, Vietnam, in 2015. He is currently pursuing the Ph.D. degree with Queen's University Belfast, UK. In 2016, he worked as a research assistant with WNDS Group, Singapore University of Technology and Design. His research interests include index modulation, machine learning, deep learning, compressed sensing, and their applications to machine-type communications.

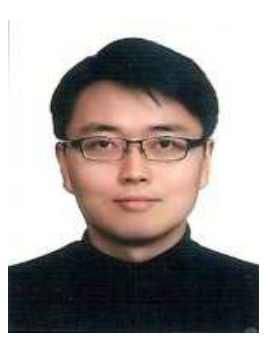

Youngwook Ko received Ph.D. in 2006 and M.S in 2002, in Electrical Engineering from Arizona State University, Tempe, AZ USA, and B.S.E. in Information and Communications Engineering from Hannam University, South Korea. After his Ph.D. he worked at Samsung for two years, as a senior researcher. In 2008, he was in Electrical and Computer Engineering at the University of Alberta, Canada. Between 2010-2013 he was with the CCSR/5GiC, University of Surrey, UK, as a senior research fellow. Since 2013, Dr. Ko works in the ECIT Institute at the Queen's University of Belfast as a Lecturer in Wireless Communications Innovation Centre. He is on the Editorial Board of the Elsevier Journal on Physical Communications and a member of the EPSRC Peer Review Associate College. His research is in the areas of multicarrier index keying, machine type communications, wireless security and vehicular communications. 